Biology of the Cell Nucleus, Institute of Molecular Genetics AS CR, Prague, Czech Republic alzbeta.darasova@img.cas.cz

Meiosis is a key process of sexual reproducing organisms and contributes to their genetic variability. The identification of new players affecting meiosis during gametogenesis could lead to revelation of new functions of chromosomal dynamics and to identification of some possible complications during meiosis that remain unexplained. It has been shown that inaccuracies during the meiotic phases result in the chromosomal aberrations and nondisjunctions, that underlie various human genetic disorders (Down's syndrome, Klinefelter's syndrome, Turner's syndrome) or lead to infertility (up to $15 \%$ of the human population). Our project focuses on the dynamics of chromosomes during gametogenesis in the eukaryotic model $\mathrm{M}$. musculus, especially on the role of vinculin (VCL) in the nucleus of mouse meiocytes. VCL is known as a cytoplasmic actin-binding protein associated with cell-cell and cell-matrix junctions. However, we observed a localization of VCL to the nuclei of primary spermatocytes in prophase I. The depletion of VCL in the primary spermatocytes has an effect of reduced fertility, premature desynapsis of homologous chromosomes in the diplotene stage and VCL also showed to be important for the exit from diplotene stage of meiosis I. My part of the project is to study VCL in primary oocytes. My aim is to answer the questions: what is the localization of VCL in prophase I oocytes? Is the involvement of VCL in prophase I oocytes similar as we observe in spermatocytes? What is the effect of VCL depletion on the progression of meiosis I in oogenesis? Are there any chromosomal aberrations? What is the fertility of these VCL-deficient females? Main methods I am using to answer these questions are: crossing mice to generate a conditional VCL knockout in mouse ovaries using the Cre lox-P system; isolation of embryonic ovaries in 15-18 dpc; phenotyping the mature ovaries of adult mice. So far, we confirmed a reduced fertility of VCL cKO females, specifically a significantly lower number of pups. Thus, we plan an in vitro fertilization assay to specify the fertility phenotype. Next, we observed a co-localization of VCL with the synaptonemal complex so we want to study the assembly and disassembly process of synaptonemal complex after depletion of VCL in primary oocytes.

Supported by the project,BIOCEV”'(CZ.1.05/1.1.00/02.0109) and "Modernization and support of research activities of the national infrastructure for biological and medical imaging Czech-BioImaging" (No. CZ.02.1.01/0.0/0.0/16_013/0001 775) funded by the European Regional Development Fund, by GACR (16-03403S and 18-19714S) and UMG/RVO: 68378050. We acknowledge the Ministry of Education, Youth and Sports of Czech Republic COST Inter-excellence internship program LTC17.

doi: http://dx.doi.org/10.7124/bc.0009DA

\section{D-2. Structural reorganization of nuclei of wheat antipodal cells during programmed cell death}

\author{
Tatiana V. Doronina $^{1}$, Inna A. Chaban ${ }^{2}$, \\ Elena M. Lazareva ${ }^{1}$ \\ ${ }^{1}$ Lomonosov Moscow State University, Moscow, Rus- \\ sia; ${ }^{2}$ Russian Institute of Agricultural Biotechnology \\ matveevatatiana.94@yandex.ru
}

The endosperm is an important part of definitive seed. It is known that endosperm cannot nor- 
mally develop in embryo sac with defective antipodal complex. The antipodal complex is located between the endosperm and the maternal tissues of ovule. The main function of antipodal cells with polytene chromosomes is synthesis of substances for the formation and protection of endosperm syncytium. The antipodal cells of Poaceae pass through consecutive stages of proliferation, differentiation and death. During their differentiation, antipodals nourish the emerging endosperm and the endosperm cellularization induces antipodal cell death. Speculated, the functioning of polyploid antipodal cells gives Poaceae an evolutionary advantage over other plants, because Poaceae is a most widespread group of plants on the Earth. Moreover, antipodal complex is a unique model for understanding the structure of polytene chromosomes. The aim of our work was to study the morphology of the nuclei of antipodal cells isolated from fertilized ovules (containing an inducer of cell death, endosperm) and unfertilized ovules (without endosperm) at different stages of programmed cell death. The work was performed on total specimens of embryo sacs isolated from fixed wheat ovules. We used methods of light microscopy (DAPI staining, acridine orange staining, Ag-Nor staining, immunocytochemical staining by the antibodies to fibrillarin, TUNEL assay) and transmission electron microscopy. We demonstrated that in fertilized embryo sacs during the differentiation, individual giant polytene chromosomes are formed in the nuclei of antipodal cells. During the death, chromosomes become more condensed and come together. Components of nucleoli segregate into the cavities of chromosomes, chromosomes become indistinguishable. Some nuclei can be strongly flattened and oblong. Dense chromatin of some nuclei may be fragmented. Then antipodal cells strongly converge and are absorbed by the endosperm. In the nuclei of antipodal cells from unfertilized embryo sacs, individual chromosomes can be detected at the stage of differentiation. During the death, chromosomes condense, in some cells, chromosomes come together, but complete integration of all chromosomes does not occur. In some nuclei, fragmentation of chromatin takes place, in others degradation of chromatin is observed in the central region of the nucleus. Segregation of the nucleolar components does not occur; nucleoli remain intact longer than the chromatin. Nuclei of antipodals from fertilized and unfertilized embryo sacs at the stage of the death are TUNEL-positive. To sum up, the morphology of the antipodal nuclei from fertilized and unfertilized ovules differs and has its own features.

doi: http://dx.doi.org/10.7124/bc.0009DB

\section{D-3. Transcription of pericentromeric tandemly repeated DNA transcripts in human preovulatory oocytes}
M. A. Dobrynin ${ }^{1,3}$, A. S. Kalugina ${ }^{3}$, N. M. Korchagina ${ }^{1}$, A. Prjibelski ${ }^{2}$, O. I. Podgornaya ${ }^{1,2}$, N. I. Enukashvily ${ }^{1 *}$.
${ }^{1}$ Institute of Cytology RAS, St. Petersburg, Rus- sia; ${ }^{2}$ St. Petersburg State University, Russia; ${ }^{3}$ ART Hospital AvaPeter-Scandinavia nie@newmail.ru

Only about 5 percent of DNA is made up of protein-coding genes; the other 95 percent is noncoding and referred as junk DNA. Most of 